\title{
Biomorphic Transportation Frameworks for Cities of the Future
}

\author{
Exploring new design framework for transportations
}

- Mint Penpisuth Wallace

Victoria University of Wellington, New Zealand

mintwallace@gmail.com

- Marc Aurel Schnabel

Victoria University of Wellington, New Zealand

marcaurel.schnabel@vuw.ac.nz

\begin{abstract}
Development in the study of city expansion, through biomorphic generation, could potentially assist in the design of a transportation system that will accommodate expansion whilst contributing to an ecologically balanced growth. This paper focuses on a section of $a$ design framework which looks at how generative systems can be implemented in an architectural design of a transportation network that will respond to the site's projected future growth. This paper suggests that by integrating biomorphic generative systems into the design process we can generate designs that respond to the surrounding context and urban growth.
\end{abstract}

Keywords: Biomorphic Algorithm, Generative Design, Processing, Urban Growth

\section{Introduction}

Rapid increases in high economic growth and employment opportunities in Bangkok have led to an influx of labour immigration, resulting in a surge of suburbanization around the metropolitan area. This unprecedented expansion of the city has resulted in the elimination of vital existing agricultural land, forests and water basins surrounding that area (Tonmanee \& Kuneepong, 2004, p. 117). The lack of flexible city planning, in terms of projected future growth, has led to an ecological imbalance causing many problems such as; the dissolution of agricultural settlements from urban sprawl, congestion in the core of the city, irrational and ever growing commuting distances, which align with the view of Constantinos Doxiadis' Ekistics theory (Doxiadis, 2005).

This paper is part of a research that proposes a transportation system developed based on two city growth theories, Doxiadis' Ekistics theory (Doxiadis, 1968) and the metabolism movement (Miles, 2013) (Figure 1). By using the two theories to understand the urban sprawl and translating them into biomorphic growth, we can attempt to control the direction of growth through physical infrastructural arrangement, topology and geometry of street and rail networks (Batty, 2008, p. 770).

We present here this first part of a design research "Design Part 1" that focus on a train station infrastructure that connects Bangkok with its neighbouring provinces in our research we employ biomorphic growth systems.

'Design Part 1' focuses on the station infrastructure design using biomorphic growth simulation though Processing language (Terzidis, 2009), which is a software sketchbook for designers that allows for simulations and visual arts coding.

The site is located in southern Lopburi in Thailand was chosen based on: the continual growth of Bangkok and the decrease of agricultural land (Tonmanee \& Kuneepong, 2004, p. 116) and the Thai Government's urban planning guideline. The proposed site has a high potential to transform into another unbalanced urbanization between urban sprawl and agricultural settlements. The programmatic imperative of a transportation infrastructure would influence the directional growth of city's network system (Doxiadis, 2005, p. 52).

\section{Problem Statement}

Whist generative design systems have been implemented before in the past, this paper questions 'how biomorphic growth models can influence and be implemented into infrastructural design!

\section{Methods}

The overall research framework for this paper is illustrated in Figure 2, with the main focus on the process of integrating biomorphic growth models to infrastructural 


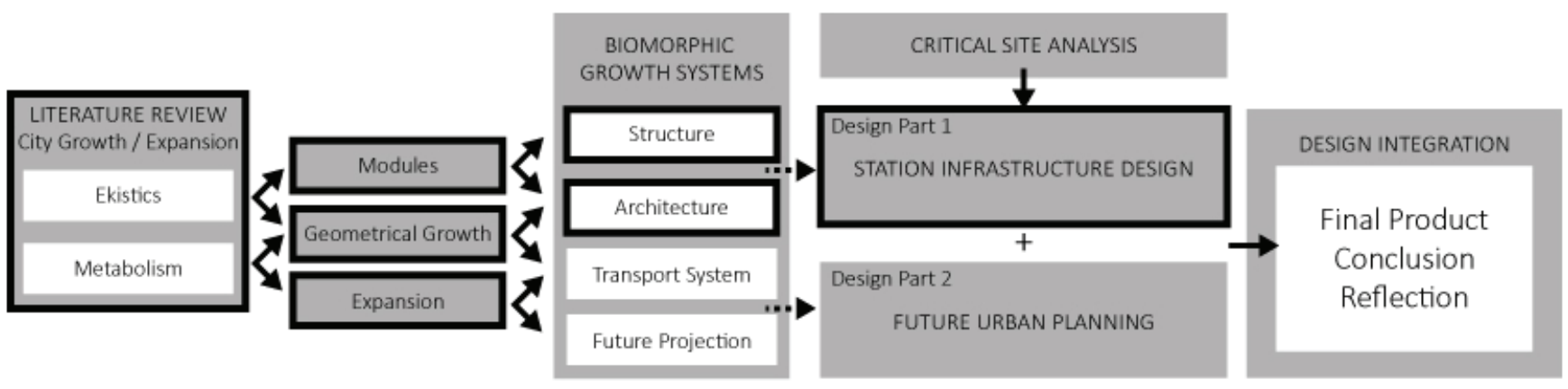

Figure 1: Research Framework of the Process and Connections for a Transportation System Development Based on City Growth Theories.

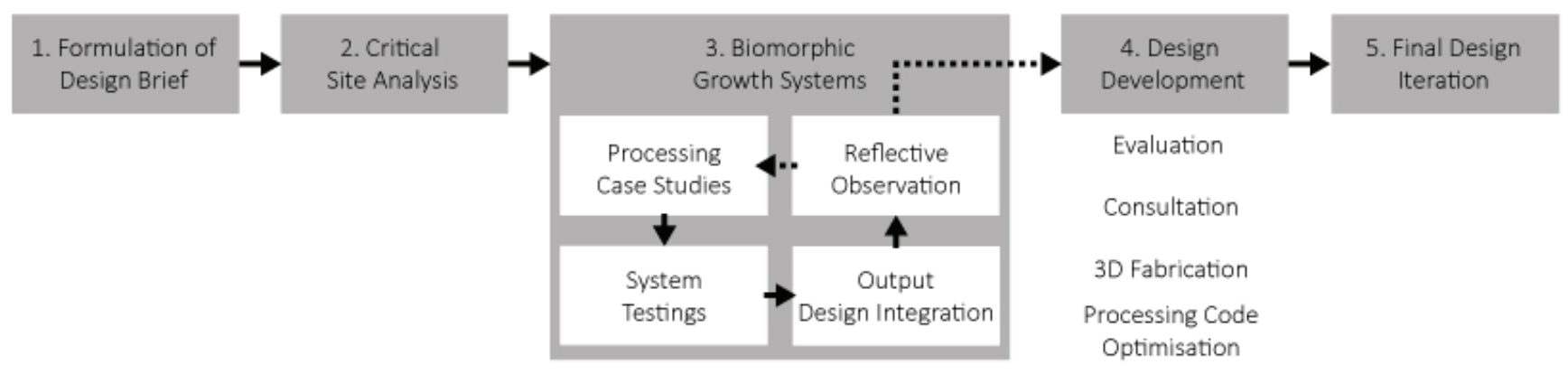

Figure 2: Framework for Integrating Biomorphic Growth Models to Infrastructural Design.

design. The study of biomorphic growth systems allows for a better understanding of how each model operate in order to evaluate the possibilities of integration with the design.

The paper commences after relevant literature reviews on city growth, focusing mainly on the Ekistics Theory (Doxiadis, 1963) and the Metabolism movement (Kuan \& Lippit, 2012). A design brief is then formulated based on critical site analysis which was carried out to provide contextual input during the design experimentation process. The literature reviews suggested that transportation systems can be viewed as biological networks which evolves over time, it follows a similar scaling effects in the networks of urban systems and cities. (Weinstock, 2011)

Development of the biomorphic growth system and growth of the optimized transportation network are generated through the systems generation process that has been adapted from "Kolb Learning Cycle" (Kolb, 2015). Experimentation on relevant case studies allows the generation of a design framework, starting with growth simulation using Processing. This stage explores the possibilities of growth mimicry from high parameter inputs (low levels of self-generations) to fully self-generated growth based on biomorphic algorithms (Lange, 2012).

A collection of different growth study types are then tested, such as Cellular Automata, Lindenmayer Systems, Recursions and Ant Colony Optimization Algorithms, which are codes that are expressed in the software.

After the experimentation, the results is then translated from Processing into vector graphics (SVG file) for 2D translation of the design, virtual 3D objects (OBJ files) or Excel data (XLS files) for calculations and data collection. Varying in the types of export materials, they are integrated into the design process at different stages.

During the Reflective Observation Stage, if the experimentation appears to be productive, it moves into the Design Development Stage. This can be measured by the flexibility of codes and integrative possibilities to the design.

The coding aspect is revisited to optimise the outcome for design integration before the Design Development Stage. However, if the results are unsatisfactory, in terms of design integrative abilities, then other growth models are tested.

The Design Development Stage consists of: evaluations, consultations, 3D fabrications and code optimisations. Each process informs the next and were revisited numerous times.

Lastly, the final design iteration is finished and waits to be implemented onto the overall urban design framework.

\section{Results}

The formulation of the design brief and the critical site analysis resulted in being a reiterative process which led to the design brief for a structural system of the high speed rail station.

The Lindenmayer system, was used as a case study following a design precedent of Asahikawa Station by Hiroshi Naito (Naito, 2013). 
After numerous testing of different Processing sketches, it is found that David King's Fractal Tree 3D (King, 2014) has potential to be developed further to match the design brief and site context. (Figure 3)

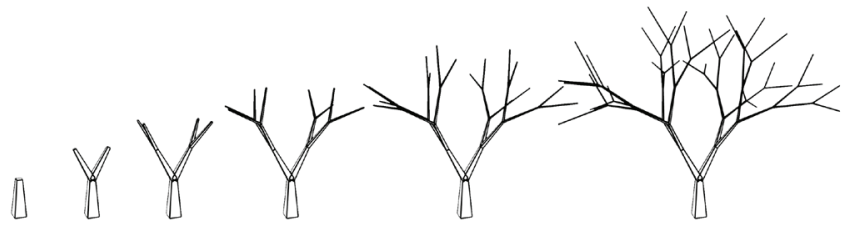

Figure 3: David King's Fractal Tree 3D in Processing.

The above sketch was studied and tweaked to test out the varying possibilities for integration into the design of the transport station. The sketch embrace the usage division of $\mathrm{Pi}$, in terms of rotation of branches, creating a balanced system for structural loads similar to that of Frei Otto's form finding techniques (Lopez, Paio, \& Sousa, 2014).

The output generated was then exported into an OBJ file via the plugin OBJExport for Processing (Louis-Rosenberg \& Zoellner, 2013) and the output was imported in Blender 3D Modelling software (http://www.blender.org). Figure 4 shows the translation of the Processing output of 3D planes to solid geometry in Blender 3D.

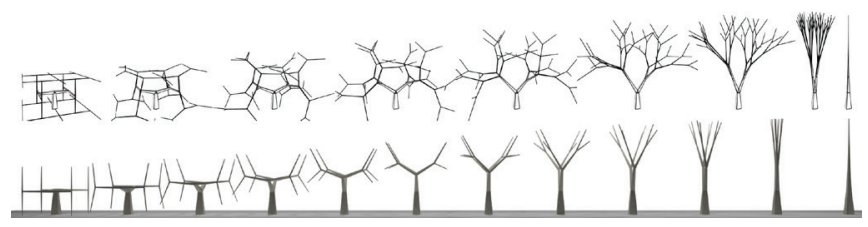

Figure 4: Framework for Integrating Biomorphic Growth Models Output from Processing to Virtual 3D Object in Blender 3D.

The core requirements of the station formulated from Stage 1 determined the basic program requirements for the station. This led to Stage 4 of the framework which focuses on design development. Over the course of one month, the Fractal Tree 3D sketch was modified and optimised, with some sections fully re-coded base on the design evaluation, consultation with a structural engineer.

The Fractal Tree 3D (FT3D) system was first integrated to the station in plan and section (Figure 5). Once the scaling of each tree was arranged for the station's basic requirement of rail systems, the code was adjusted to accommodate the height and width requirements for high-speed train tracks.

The platform for the high-speed train and the regular train were merged based on a design evaluation of the circulation which led to further re-coding of the FT3D sketch. Spheres were generated at each branch separation (Figure 6) to allow for an easier transition to the platform design.

At this stage, the FT3D demonstrated the possibility to be integrated into the design as not only the structure for the platform but also the underlying structure for the canopy.

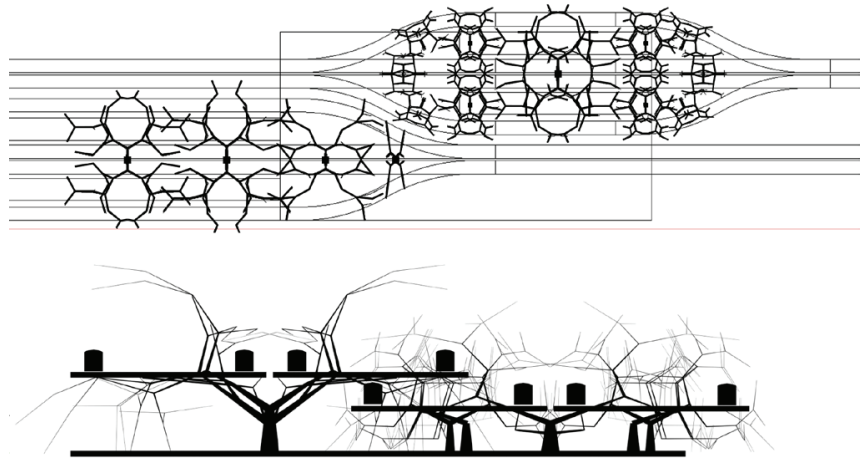

Figure 5: Plan \& Section Integration.

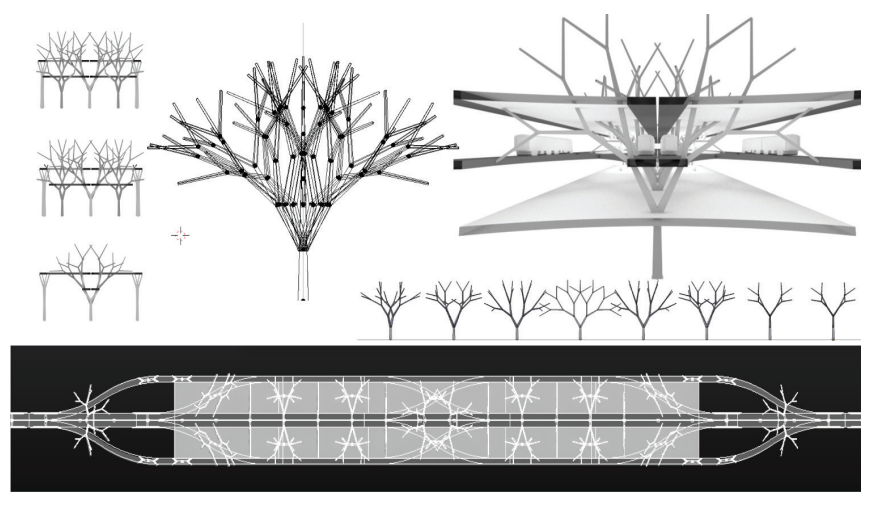

Figure 6: Sectional Studies for Vertical Relation of the Tree Structure and Platform and Transition of Tree Structure in Elevation and Plan.

After several consultations with a structural engineer, the FT3D output was separated into two materials, reinforced concrete for heights lower than the platform and steel for the rest of the structure the main material for the canopy of the station.

Shape iterations of the concrete base were then tested through a fabrication using an ABS 3D printer. Several geometric modifiers were used with the base geometry of the FT3D sketch output. Figure 7 illustrates some examples of this experimentation with sub-division surface modifier to strengthening the base and remove excess geometry. The bone modifier reduce the outer surface to only the core structure.

Fabrication process and the consultations informed the design development towards the utilisation of the subdivision surface modifier to strengthen the output delivered from Processing.

Growth of the optimised FT3D output is then translated according to the previous modifications and materials configurations (Figure 8).

Generations of the canopy were developed through the connection of each branch as they gradually grow. Materials specifications and capabilities were considered in this process to ensure a practical design.

The canopy was divided from each connection of the structure to create space for the panels that would 
accommodate the form generated. Figure 9 shows the relationship between the elements of the whole design from the concrete base structure to the steel canopy structure in relation to the canopy and its framing.

Circulation of the programmes on the platforms was then added between the ground level, regular train platform, and high speed train platform.

The final design iteration (Figure 10) is an example of one of the possibilities that this framework can produce. The infrastructure design framework is ready to correspond to the result of 'Part 2 Future Urban Planning'.

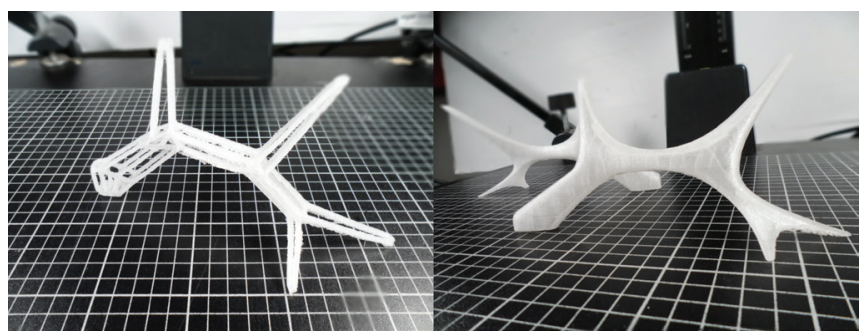

Figure 7: 3D Printing Results with the Bone Modifier (left) and Subdivision Surface Modifier (right).

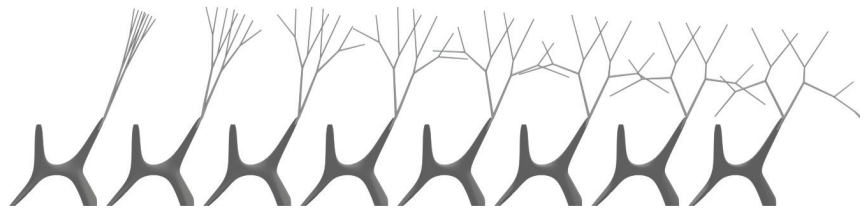

Figure 8: Optimised Result of the Translated Output from FT3D.

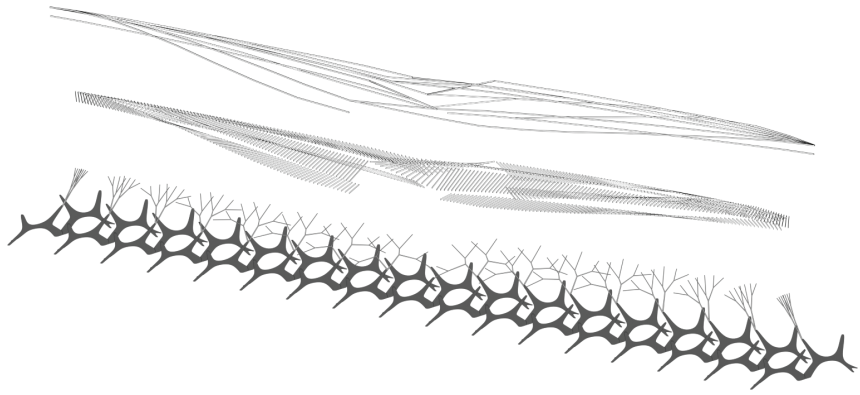

Figure 9: Exploded Axonometric of the Canopy and Structure.

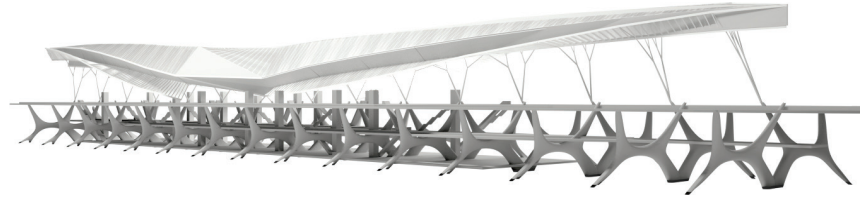

Figure 10: Final Design Iteration.

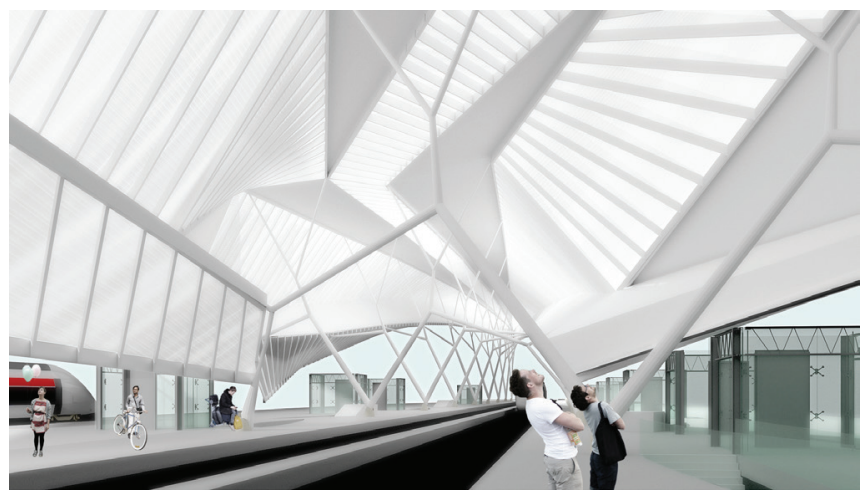

Figure 11: Interior Rendering of Canopy Structure.

\section{Discussion}

Exploration of biomorphic growth models using Processing was an excellent choice for this paper as the software was designed for artists with basic knowledge of computer coding, the software allows for basic and advance simulations to be generated and exported for further development.

This method relies heavily on understanding and testing many case studies in order for the evaluation of each sketch to be informed. The availabilties of Processing sketches and tutorials from the online communities played a great role in the development of the sketch to accommodate the design.

Throughout the design process, the self-evaluation and consultation sessions were helpful in term of assessing the output from the biomorphic growth models to the design.

Fabrication of the elements using the 3D printer allows for a better understanding of scale as well as the strength of each iteration. Further investigation into this process can lead to even more optimisation of the outcome.

Generation process of the canopy structure from the tree branches were manually created through a form finding process. The results (Figure 11) generated a design influenced by the L-system. With the form finalised, the procedure can be develop into an automatic process for future use in Part 2 of the research.

In conclusion, a biomorphic growth model can be implemented to infrastructural design using a framework which investigates and evaluates each sketch by the compatibility to structural elements. The understanding of the relationship between the growth model coding and design integration appears to be a key to a seamless integration of biomorphic growth model and infrastructural design.

\section{References}

Batty, M. (2008). The Size, Scale, and Shape of Cities. Science, 319(5864), 769-771. 
Doxiadis, C. A. (1963). Architecture in transition. London Hutchinson.

Doxiadis, C. A. (1968). Ekistics: an introduction to the science of human settlements. London: Hutchinson.

Doxiadis, C. A. (2005). Towards an ekistic theory. Ekistics, 72(430-435), 39-66.

King, D. (2014). Fractal Tree 3D. 2015, from http://www. openprocessing.org/sketch/153474

Kolb, D. A. (2015). Experiential learning: experience as the source of learning and development. Upper Saddle River, New Jersey: Pearson Education Ltd.

Kuan, S., \& Lippit, Y. (2012). Kenzo Tange: architecture for the world. London; Baden: Lars Müller.

Lopez, J., Paio, A., \& Sousa, J. (2014). Parametric Urban Models Based on Frei Otto's Generative Form-finding Process.
Louis-Rosenberg, J., \& Zoellner, M. (2013). Nervous Systems | Tools | OBJExport. 2015, from http://n-e-r-v-o-u-s.com/tools/ obj/

Miles, M. (2013). Metabolism: A Japanese Modernism. Cultural Politics, 9(1), 70-85. doi: 10.1215/17432197-1907181

Naito, H. (2013). Hiroshi Naito : from protoform to protoscape: Tokyo-to Minato-ku : TOTO Shuppan.

Terzidis, K. (2009). Algorithms for visual design using the Processing language. Indianapolis, IN: Wiley Pub.

Tonmanee, N., \& Kuneepong, P. (2004). Impact of land use change in bangkok metropolitan and suburban areas. Planning Metropolitan Landscapes, 115.

Weinstock, M. (2011). The metabolism of the city. Architectural Design, 81(4), 102-107. 